Instituto Internacional de Investigación y Desarrollo Tecnológico Educativo INDTEC, C.A.

DOI: https://doi.org/10.29394/scientific.issn.2542-2987.2017.2.3.13.247-259

OAI-PMH: http://www.indteca.com/ojs/index.php/Revista Scientific/oai

\title{
Huertos Escolares como Recurso Didáctico para el Desarrollo Sustentable de la Comunidad
}

\author{
Autora: María Inmaculada Hernández \\ Universidad Pedagógica Experimental Libertador, UPEL \\ immac12467@gmail.com \\ Barinas, Venezuela
}

\section{Resumen}

La presente investigación tiene como propósito implementar huertos escolares como recurso didáctico para el desarrollo sustentable hacia una cultura ambientalista, en la Escuela Técnica Agropecuaria Nacional "Francisco Tamayo" de la parroquia Barinitas Municipio Bolívar estado Barinas. Tiene como objetivo fundamental dar una alternativa viable y sustentable desde la escuela hacia la comunidad. La misma se encuentra bajo el enfoque cualitativo, a través del método investigación-acción participante. El diseño metodológico está estructurado en cinco etapas: (1) Diagnóstico; (2) planificación; (3) Ejecución; (4) evaluación; (5) Sistematización. Los informantes claves estarán representados por tres docentes relacionados a la parte agrícola, tres estudiantes de tercer año sección $A$, y un Directivo quien dará la información pertinente que servirá de base para la planificación y ejecución de las estrategias. Las técnicas de recolección de información serán la entrevista a profundidad y la observación de documentos. Las técnicas de análisis de la información fueron la categorización, triangulación e interpretación. didáctico.

Palabras clave: desarrollo sustentable; huerto escolar; recurso 


\section{School Vegetable Patch as a Didactic Resource to the Sustainable Development of the Community}

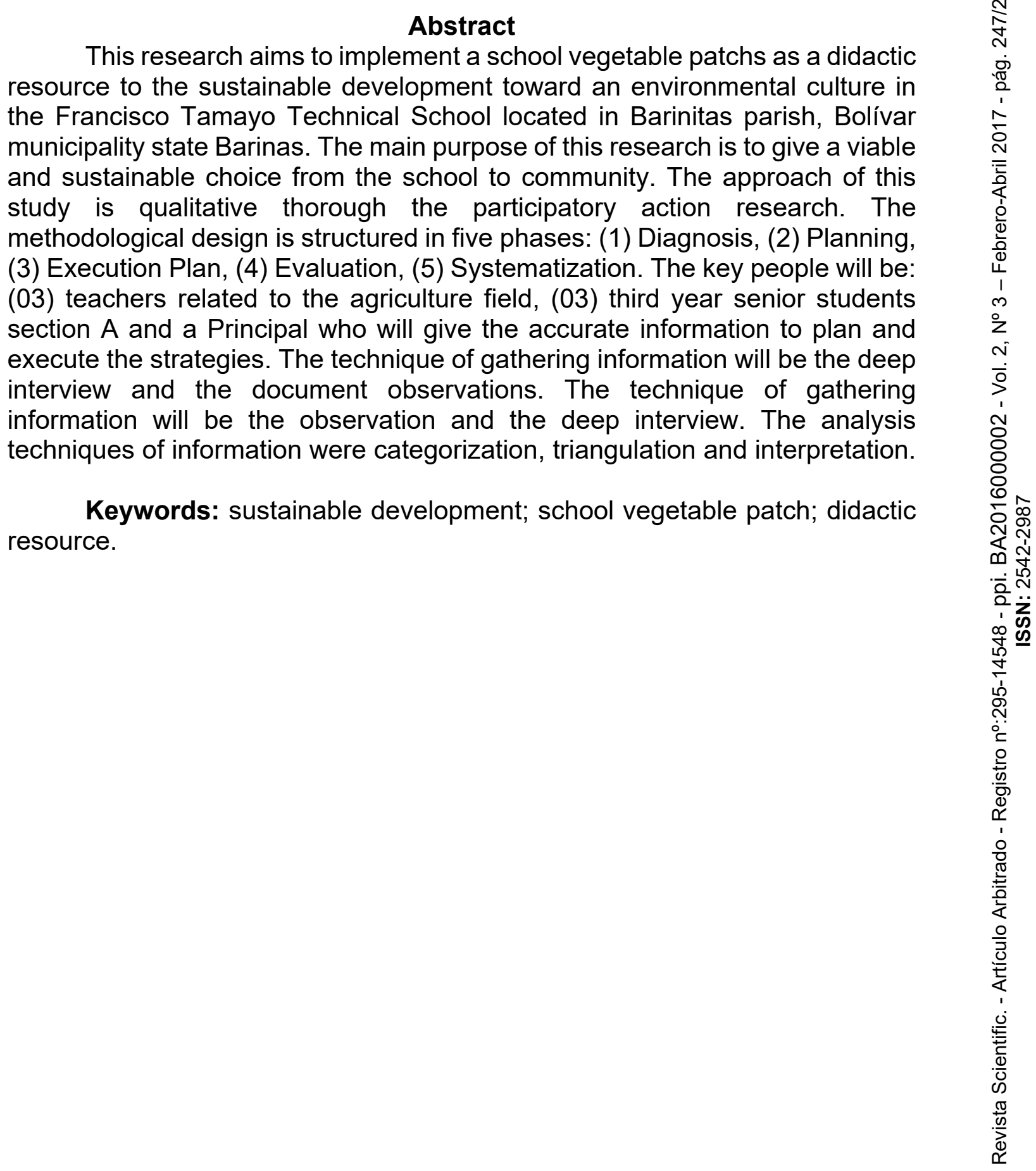

Date Received: 11-09-2016

Date Acceptance: $17-10-2016$ 


\section{Introducción}

El hombre desde sus inicios de la vida en el planeta, se vio en la necesidad de dominar las fuerzas de la naturaleza para obtener su alimento, vivienda, calefacción y vestido. Desde entonces, ha empleado los recursos naturales para adquirir la energía necesaria que garantizara la sobrevivencia de la especie humana, aun cuando tenía un escaso conocimiento tecnológico la lucha racional fue siempre por la subsistencia. Hoy después de varias centurias la lucha sigue siendo igual, con una particular diferencia, los recursos naturales se encuentran cada vez más amenazados ante tanta contaminación, la garantía de acceder a los productos de la naturaleza se torna en entredicho. En ese sentido, se considera que el desarrollo de la humanidad se caracteriza por ser un proceso multidimensional, determinado por características del contexto histórico social-cultural frente al mundo acelerado debido al proceso de globalización.

Sobre las bases de las consideraciones de la autora, es preciso reflexionar porque justamente evidencia que los países proveedores de materia prima en lugar de planificar sus modelos de desarrollo, en especial los relacionados con el sector productivo hacia la reducción del impacto ecológico, en función de cambiar los modelos existentes, por aquellos que causen menos impacto negativo al ambiental, han aprovechado los recursos naturales causando daños irreversibles a los contextos naturales y urbanos. Se denota la escasa planificación del crecimiento urbano, el excesivo uso de herbicidas y contaminantes que generan daños a los alimentos consumibles.

Dentro de estas consideraciones se señala, el Banco Mundial en su objetivo 7, plantea:

El desarrollo sostenible reconoce que el crecimiento debe ser inclusivo y al mismo tiempo ambientalmente racional para poder reducir la pobreza y generar prosperidad, tanto para quienes viven en el planeta en el presente como para las futuras generaciones... La tierra sigue degradándose, se 
pierden bosques y las especies están en peligro de extinción a medida que las emisiones de carbono continúan cambiando el ambiente en el que vivimos. Con apoyo del Banco Mundial, más países están abordando las relaciones entre tierra, bosques, agua y seguridad alimentaria de una manera más integral y sostenible (Banco Mundial, 2015, pág. 26).

Dicho lo anterior, la idea central de esta investigación subyace en la necesidad de una nueva concepción del modelo productivo que valore el impacto ambiental, la educación de valores ambientales en la comunidad y la huella ecológica que deja esta generación y su incidencia a futuro. Dentro del marco de estas ideas que rodean a esta situación planteada, la educación está llamada a jugar un papel fundamental, por cuanto al educar se cambia patrones de comportamiento ante los recursos naturales, modos de vida, enfocadas en un cambio de actitudes y valores ambientales, siendo las actividades de cultivo de la tierra desde la escuela aprovechando los espacios naturales.

De esta forma, el presente estudio afronta el desarrollo sustentable a través de la integración escuela-comunidad, utilizando recursos desde el punto de vista ecológico, es decir nuevos métodos que les permitan desarrollar técnicas de producción, y al mismo tiempo minimicen el impacto ambiental. Para ello, se hace necesario cambio de comportamiento, valores y conductas mediante actividades fomentadas por las instituciones educativas, para que sean puestas en práctica en los hogares; con lo cual se garantice a las presentes y futuras generaciones un uso racional de los recursos renovables.

Por su parte, la Escuela Técnica Francisco Tamayo no escapa a esta realidad y en la práctica se observa la escasa existencia de huertos escolares como estrategia didáctica que promuevan la sostenibilidad de los recursos naturales; dado que la aplicación de este conocimiento es una opción favorable en la conservación de la tierra, contribuyendo adecuadamente con 
la solución y uso adecuado del suelo, mediante la realización de un trabajo mancomunado y compartir experiencias a través del contacto directo con todo los miembros involucrados.

En tal sentido, se puede considera que a través de la siguiente investigación se quiere efectuar un trabajo de promoción en la realización de los huertos escolares como recurso didáctico para el desarrollo sustentable de la comunidad y estudiantes de 3er año, sección "A" Escuela Técnica Francisco Tamayo, Municipio Bolívar; motivado a que se aspira integrar a la comunidad educativa como parte importante para el desarrollo de la institución escolar.

\subsection{Objetivos de la Investigación}

\subsubsection{Objetivo General}

Promover los huertos escolares como recurso didáctico para el desarrollo sustentable de la comunidad y estudiantes de 3er año, sección "A" Escuela Técnica Francisco Tamayo, Municipio Bolívar.

\subsubsection{Objetivos específicos}

Diagnosticar la implementación de los huertos escolares como recurso didáctico para el desarrollo sustentable de la comunidad y estudiantes de 3er año, sección "A" Escuela Técnica Francisco Tamayo, Municipio Bolívar.

Plantear el desarrollo de huertos escolares como recurso didáctico hacia el desarrollo sustentable de la comunidad con los estudiantes de $3 \mathrm{er}$ año, sección "A" Escuela Técnica Francisco Tamayo, Municipio Bolívar.

Contribuir a la socialización, formación de actitudes y valores del desarrollo sustentable de la comunidad y estudiantes de 3er año, sección "A" Escuela Técnica Francisco Tamayo, Municipio Bolívar, considerando los huertos escolares como recurso didáctico. 
Sistematizar la experiencia con los huertos escolares como recurso didáctico hacia el desarrollo sustentable de la comunidad y estudiantes de 3er año, sección "A" Escuela Técnica Francisco Tamayo, Municipio Bolívar.

\section{Marco Referencial}

\subsection{El Huerto Escolar}

Para definir lo que engloba el concepto de huerto, Según Cáseres, (2005): "el huerto escolar también se le conoce como huerto para aprender distinguiéndose este tipo de huerto por ser una parcela pequeña de tierra que la atienden pocas personas o un grupo" (pág. 58). En este tipo de producción, pueden predominar las hortalizas, pero incluye frutos menores y ornamentales. Requiere la colaboración de todos para las labores iniciales más rudas como la preparación del terreno y su cercado; en las etapas siguientes se comparten las labores de siembra, cultivo y cosecha.

Una definición más amplia es:

Un sistema de producción de tipo agrícola y/o forestal, que ocupa un área, generalmente cercana a la vivienda, donde se presenta la asociación intensiva y permanente de diversas leñosas multipropósito, frutales y diversas hortalizas y legumbres, además de la cría de animales domésticos (Ospina 2008, pág. 47).

Por lo comentado, se puede decir que el huerto familiar, al igual que la mayoría de las tecnologías agroforestales, tiene su origen, en culturas agroforestales; puede afirmarse que el huerto constituye una pieza clave en el origen de la agricultura. De esta manera, que cultivar un huerto ecológico es algo más que obtener verduras, frutas y hortalizas de manera natural, dado que los frutos más preciados que se consiguen con su cultivo son los beneficios para la propia salud. 


\subsection{Beneficios de una Huerto Escolar}

El trabajo de los huertos escolares es de importancia, para ello, Bueno (2009), considera que:

- Mejora la calidad de la alimentación con la inclusión de verduras.

- Disminuye los costos, generando ingresos con la venta de verduras frescas o algunos productos procesados como salsas y dulces.

- Genera y promueve actitudes positivas hacia la autogestión comunitaria y de la institución.

- Aprovecha del tiempo libre de los niños y niñas.

- Contribuye a la inclusión de las personas con dificultades físicas o mentales al poder participar en las actividades del huerto haciéndolas sentirse útiles y porque no contribuir a su bienestar.

- Fortalece el ambiente, contribuyendo al mantenimiento de la flora y fauna autóctona.

\subsection{Paradigma de la Investigación}

La naturaleza de la investigación, se ubicará en un estudio de características dentro del paradigma cualitativo; puesto que el mismo permitirá hacer un análisis de la situación social, cultural y económica, así como las habilidades y destrezas de los actores involucrados, para hacerle frente a la necesidad de huertos escolares como recurso didáctico en el desarrollo sustentable de la comunidad.

Por ello, se tomará en consideración lo mencionado por Hurtado y Toro (2006), al comentar que a través de él se "descubren las particularidades de un elemento y se interpretan sus características en el contexto específico" (pág. 21). Por esta razón, en el paradigma cualitativo el propósito consiste en describir e interpretar sensiblemente la vida social y cultural de quienes participan; tal como se efectuará en la investigación de promover los huertos escolares como recurso didáctico para el desarrollo sustentable de la 
comunidad y estudiantes de 3er año, sección "A" Escuela Técnica Francisco Tamayo, Municipio Bolívar.

\section{Metodología}

\subsection{Diseño de la Investigación}

De acuerdo la revisión de la investigación; así como a su propósito se ubicará en el tipo de Investigación Acción Participativa, la cual para realizar este tipo de estudio se:

Combina dos procesos, el de conocer y el de actuar, implicando en ambos a la población cuya realidad se aborda. Al igual que otros enfoques participativos, la IAP proporciona a las comunidades y a las agencias de desarrollo un método para analizar y comprender mejor la realidad de la población (sus problemas, necesidades, capacidades, recursos), y les permite planificar acciones y medidas para transformarla y mejorarla. Es un proceso que combina la teoría y la praxis, y que posibilita el aprendizaje, la toma de conciencia crítica de la población sobre su realidad, su empoderamiento (Eizagirre y Zabala, 2004, pág. 25)

En este estudio se generará un proceso de reflexión acerca del uso racional de recursos, implementando técnicas ecológicas y utilizando elementos del entorno para realizar las actividades agrícolas, es por ello que en concordancia con lo expresado, se cumplirá con las siguientes fases propuestas por Buendía, Colás y Hernández (2008) que se estructura en cinco (05) etapas de acción que se parafrasean, a continuación:

Etapa I Diagnóstico. Se procede a reconocer de forma clara la problemática presentada, a través de la identificación de las necesidades y problemas. El diagnóstico participativo se realizará a través de la entrevista a profundidad, sustentada en un guion de entrevista para la recolección de información, se usarán algunas técnicas como la observación participante. 
Etapa II Planificación. Abarcar el diseño de un plan de trabajo con la finalidad de facilitar la acción, en este caso, se desarrollarán actividades y acciones enmarcadas en técnicas, forma y momento de aplicación adecuadas para la solución a la problemática presentada por la población en estudio.

Etapa III Ejecución. En esta etapa se desarrollan las acciones planificadas dirigidas a ejecutar acciones para la construcción de los huertos.

Etapa IV Evaluación. En la misma se valorarán las acciones contentivas en el plan de acción. Cada actividad se evaluará de manera continua con la finalidad de ir reflexionando sobre los procesos emprendidos e ir generando los cambios requeridos o consolidando los logros obtenidos; apoyándose en la realización de la triangulación para conocer los criterios de los sujetos e investigador.

Etapa V Sistematización. Las experiencias se constituirán de todo el proceso de trabajo, para luego ser planificadas y presentadas como una alternativa de solución viable tal como se expresará en la categorización.

\subsection{Participantes (informantes claves)}

Con relación con la investigación, se utilizarán como informantes claves tres (03) docentes relacionados con el trabajo agrícola dos (02) estudiantes de tercer año "A" y un (01) directivo; esto se efectúa tomando en consideración las características del estudio a través de la revisión en primer momento por medio de la observación donde se podrá de manifiesto la verificación de las necesidades de los involucrados, para luego hacer una revisión de los archivos llevados en la institución escolar, con la finalidad de efectuar la selección de los informantes.

\subsection{Técnicas e Instrumento de la Información en la Investigación}

El análisis de datos cualitativos es un largo proceso que se inicia con la recolección de las informaciones y se desarrolla en algunos momentos, 
conjuntamente con el cuaderno de notas. Interpretar exige atención a los detalles, identificar, comparar, relacionar e ir de las partes al todo y del todo a las partes, es un movimiento constante de los datos a la reflexión y de la reflexión a los datos.

Para esta investigación se aplicarán como técnica la entrevista en profundidad y la observación de documentos; mientras que el instrumento será un guion de entrevista, enfocado en acciones que muestren construcciones teóricas derivadas de la práctica misma, debido a que se adaptan mejor a los objetivos del estudio. Por su parte, la entrevista en profundidad son encuentros cara a cara, abiertos no directivos, entre el entrevistador y los informantes, dirigidos hacia la comprensión de las perspectivas que tienen los informantes de su vida, experiencias y situaciones.

\subsection{Validez de la Investigación}

En este sentido, la validez de contenido se logra a través de la técnica juicio de expertos, conformado por tres (3) profesionales de alta trayectoria, especialistas en: Metodología de la Investigación y Educación Ambiental; todo ello implica que se efectuará la revisión de los aportes dado por lo sujetos de estudio, el cual se efectuará a través de actas informativas.

\subsection{Confiabilidad}

La confiabilidad constituye condiciones indispensables que deben mostrar cualquier instrumento de recolección de información, para que el producto que se obtenga del mismo goce de aceptación de la comunidad científica. Respecto a la confiabilidad, Ruiz (2009), plantea que esta se evidencia cuando el "instrumento al ser aplicado en diferentes miembros de la población arroja resultados más o menos similares" (pág. 125).

Luego se van tomando los códigos directamente de la entrevista y se utilizan sustantivos, verbos o palabras naturales del relato o texto original. 
Después se van estableciendo relaciones entre conceptos, categorías, propiedades. En los estudios realizados por medio de investigaciones cualitativas, que, en general, están guiados por una orientación sistémica, hermenéutica, fenomenológica, etnográfica y humanista, la confiabilidad está orientada hacia el nivel de concordancia interpretativa entre diferentes observadores, evaluadores o jueces del mismo fenómeno.

\section{Resultados y Conclusiones}

Los resultados y conclusiones no se presentan ya que se encuentran en análisis de estudio. Sin embargo, se hace referencia a algunas actividades enfocadas hacia la ejecución del plan de acción. Entre ellas se destacan:

- Elaboración de folletos y carteles para promover el cambio de actitud en los docentes y estudiantes relacionadas con la elaboración de huertos.

- Propiciar el intercambio de experiencias y saberes para establecer oportunidades en la elaboración de huertos escolares.

- Utilización de técnicas agrícolas sustentables.

\section{Referencias}

Banco Mundial (2015). Un enfoque integral sobre la sostenibilidad medioambiental. Resolución 66/288 de la Asamblea General de Naciones Unidas.

Buendía, I., Colás, P. y Hernández, F. (2008). Métodos de Investigación en Psicopedagogía. (3era Edición). Madrid, España: McGraw-Hill.

Bueno, G. (2009). Elementos de Control Sanitario. Publicaciones Universidad Arturo Michelena. Estado Carabobo.

Cáseres, W. (2005). Mantenimiento y Mejoramiento en los cultivos Cooperativos. El Estudio y la Actividad Humana Madrid: McGraw-Hill. 
Eizagirre, E. y Zabala, N. (2004). Investigación-acción participativa (IAP). Editorial Fondo de Cultura Económica. México.

Hurtado, F., y Toro, A. (2006). Introducción a la Metodología de la Investigación en Técnicas de Administración y de Conocimientos. Editorial Trillas, 5ta. Edición. México.

Ospina, A (2008). El Huerto Familiar. Las nuevas Estrategias de Planificación. Editorial ROMOR. Caracas, Venezuela.

Ruiz, T. (2009). Estudios e Investigaciones Científica. Revista Cuadal. Buenos Aires. 


\section{María Inmaculada Hernández}

e-mail: immac12467@gmail.com

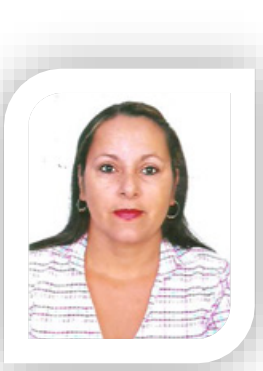

Nacida en el Municipio Pedraza Del Estado Barinas, Venezuela. Cursa estudios de magister en Educación Ambiente $Y$ Desarrollo en la Universidad Pedagógica Experimental Libertador Instituto Del Mejoramiento Profesional Del Magisterio núcleo académico barinas. Técnico superior universitario en contaduría egresada de la universidad experimental de los llanos Ezequiel Zamora (2002). Licenciada en educación mención geografía e historia (2009). A nivel universitario se ha desempeñado en el área de la docencia en la universidad bolivariana de Venezuela desde el 2009, 2012.

Actualmente se desempeña como docente de aula en la institución educativa Escuela Técnica Agropecuaria Nacional Francisco Tamayo.

El contenido de este manuscrito se difunde bajo una Licencia de Creative Commons ReconocimientoNoComercial-Compartirlgual 4.0 Internacional 